\section{A) Check for updates}

Cite this: Analyst, 2021, 146, 4873

\title{
Electrochemical operational principles and analytical performance of Pd-based amperometric nanobiosensors $\uparrow$
}

\author{
Y. E. Silina, (D) $* \neq^{a}$ N. Apushkinskaya, (D) ${ }^{a}$ N. V. Talagaeva, ${ }^{b}$ M. G. Levchenko ${ }^{b}$ and \\ E. V. Zolotukhina (D) $* \mathfrak{t}^{\mathrm{b}, \mathrm{c}}$
}

Palladium nanoparticles (Pd-NPs) have been approved as an effective catalyst for hydrogen peroxide decomposition which is released during specific enzymatic reactions. However, the general operational principles and electrochemical performance of Pd-NPs-based nanobiosensors have been poorly exploited. Here, the electrochemical behavior of oxidase-associated peroxide oxidation co-catalysis of the modelled microanalytical system based on screen-printed electrodes modified by electroplated PdNPs as an electrocatalyst, glucose oxidase (GOx) or alcohol oxidase (AOx) as a bioreceptor and the ionomer Nafion as a polymeric binding agent was studied in detail. The impact of palladium surface oxides and adsorbed oxygen on the activity and product selectivity in an oxidase type of nanobiosensor was ascertained. To avoid $\mathrm{PdO}$ and oxygen electroreduction affecting the entire amperometric response of Pd-NPs-based nanobiosensors, a special two-step polarization procedure was proposed. Under the established electrochemical conditions, Pd-NPs-based nanobiosensors with encapsulated oxidases showed a wide dynamic range towards selective bioanalyte detection, excellent basic line stability, accuracy and resistance to the presence of interfering electrochemical species. This work can serve as a guideline for the search and validation of operational principles of novel biosensors based on nanoparticles.

Received 16th May 2021 Accepted 17th June 2021

DOI: 10.1039/d1an00882j rsc.li/analyst ing the enzymatically produced product $\left(\mathrm{H}_{2} \mathrm{O}_{2}\right)$ can be mentioned as an example. The amount of produced hydrogen peroxide will be proportional to the analyte concentration in a sample. Typically, redox mediators (e.g. Prussian Blue and Meldola Blue) can be employed to facilitate electron-transfer between the bioreceptor and transducer, and to decompose the formed hydrogen peroxide via electrocatalytic redoxreaction. ${ }^{1-4}$ However, some redox mediators (electrocatalysts) used in amperometric glucose or alcohol sensitive biosensors are prone to mechanical instability that leads to their leakage from the electrode surface and degradation of the signal, insufficient sensor sensitivity and selectivity, and low repeatability and accuracy. ${ }^{5}$

As an alternative solution, nanoparticles (NPs) of noble metals have been explored in amperometric biosensor development. $^{6-8}$ They are biocompatible, mechanically and chemically stable, they exhibit advanced catalytic properties, and their synthesis is environmentally friendly and often can be conducted in a one-step/one-pot manner. ${ }^{9-11}$ More importantly, NPs of noble metals in electrochemical biosensors act directly as electrocatalysts in the oxidation/reduction reaction of the co-products (e.g. hydrogen peroxide) formed during biochemical reactions. ${ }^{12,13}$

\footnotetext{
${ }^{a}$ Institute for Biochemistry, Zentrum für Human und Molekularbiologie (ZHMB), Campus B 2.2, University of Saarland, 66123 Saarbrücken, Germany.

E-mail:yuliya.silina@uni-saarland.de,yuliya.silina@gmx.de

${ }^{b}$ Institute of Problems of Chemical Physics RAS, 1 Ac. Semenov avenue, Chernogolovka, 142432 Moscow region, Russia. E-mail: zolek@icp.ac.ru ${ }^{c}$ Moscow Institute of Physics and Technology, 9 Institutskiy per., Dolgoprudny, 141700 Moscow region, Russia

$\dagger$ Electronic supplementary information (ESI) available. See DOI: 10.1039/ d1 1 an00882j

$\$$ Authors with equal contribution.
} 
A wide spectrum of electrochemical nanobiosensors with NPs of noble metals as electrocatalysts was introduced. ${ }^{14-16}$ However, the working electrochemical conditions were optimized and validated exclusively for Pt-NPs, Au-NPs and AgNPs-based amperometric biosensors. ${ }^{17-21}$ To the best of our knowledge, almost no attempts were made towards the establishment, validation and systematization of the electrochemical system parameters of Pd-NPs-based nanobiosensors.

In our previous studies, we introduced a novel fabrication approach to produce one-step designed tailored enzymatic nanobiosensors based on Pd-NPs followed by their thorough standardization. ${ }^{5,21}$ According to this approach, the end-structure of one-step designed nanobiosensors represents a structural model mainly composed of an enzyme incorporated in metal-polymer scaffolds. ${ }^{22}$ This nanocapsular structure provides on demand controlled release of enzymes (in the presence of an analyte). However, the electrochemical operational principles and guidelines of these novel one-step designed Pdbased amperometric nanobiosensors have been poorly exploited. Meanwhile, specifically, the used working conditions (applied potential, duration of polarization, read-out detection mode, etc.) will affect the performance of the onestep designed Pd-NPs-based nanobiosensors. Thus, in some studies Pd-NPs-based systems were not recommended to be operated in a low potential range (from 0 to $-0.4 \mathrm{~V}$ ) due to oxygen electrodesorption. ${ }^{23}$ In contrast, electrochemical tuning of the enzymatic activity of Pd-NPs-based biosensors towards alcohol detection in fermentation media under very low applied potentials (from -0.02 to $-0.32 \mathrm{~V}$ ) has recently been reported. ${ }^{24}$

Hence, to avoid any misinterpretation of the obtained electrochemical results supplied by the one-step produced PdNPs-based nanobiosensors, a detailed study on the processes affecting their responses as well as the development of general working principles and operational guidelines are highly necessary.

Here, we conducted a systematic study on the operation of one-step designed Pd-NPs-based nanobiosensors under the complex conditions of a few parallel electrocatalytic reactions. The obtained results were confirmed by a series of advanced analytical techniques, i.e. scanning electron microscopy, laser desorption ionization mass spectrometry, RAMAN spectroscopy, cyclic voltammetry, multi-step amperometry and oxygen minisensor studies. More specifically, the novelty of this study relies on the established mechanism underlying the electrochemical response of one-step designed Pd-NPs-based amperometric nanobiosensors (i); the developed multi-step operational read-out platform (ii); and a novel design of PdNPs-based nanobiosensors being operated at anodic potentials which allowed extending the linear response towards glucose detection (iii).

The practical merit of this work is in the development of a time-, cost-effective and simple methodology that can significantly improve the read-out of Pd-NPs-based nanobiosensors and permit their rational design.

\section{Experimental part}

\subsection{Materials and methods}

$30 \% \mathrm{H}_{2} \mathrm{O}_{2}, \mathrm{EtOH}, \mathrm{BuOH}$, glucose, $\mathrm{AgCl}, \mathrm{K}_{4}\left[\mathrm{Fe}(\mathrm{CN})_{6}\right], \mathrm{K}_{3} \mathrm{Fe}$ $(\mathrm{CN})_{6}, \quad \mathrm{FeCl}_{3}, \quad \mathrm{H}_{2} \mathrm{PdCl}_{4}, \quad 25 \% \quad \mathrm{NH}_{4} \mathrm{OH}, \quad \mathrm{Pd}\left(\mathrm{NH}_{3}\right)_{4} \mathrm{Cl}_{2}$, $\mathrm{K}_{4}\left[\mathrm{Ag}_{2}(\mathrm{CN})_{6}\right],\left(\mathrm{NH}_{4}\right)_{2} \mathrm{HPO}_{4}$, and $\mathrm{Na}_{2} \mathrm{HPO}_{4} \cdot 12 \mathrm{H}_{2} \mathrm{O}$ (99\% purity) were obtained from Sigma Aldrich. Nafion ${ }^{\circledR 117}$ solution in a mixture of aliphatic alcohols and water, glucose oxidase, GOx, from Aspergillus Niger (EC 1.1.3.4, type VII, $403 \mathrm{U} \mathrm{g}^{-1}$ solid), and alcohol oxidase, AOx, from Pichia pastoris (EC 1.1.3.13, 32 $\mathrm{U} \mathrm{mg}^{-1}$ ), were received from Merck (Darmstadt, Germany). Pdwire, Ag-wire and Pt-wire (99\%, Sigma Aldrich) were used as the anodes for the preparation of palladium nanoparticles (PdNPs), silver nanoparticles (Ag-NPs) and Prussian Blue (PB).

DRP-110DGPHOX screen printed electrodes (SPEs) were obtained from DropSens (Llanera, Spain). Each sensor consisted of a carbon working electrode (WE) modified with a layer of graphene oxide (referred to as SPE/GO), a carbon counter electrode, and a silver reference electrode. Organicfree, deionized water (DI) was generated with an Elga PureLab (Celle, Germany) water purification system.

\subsection{Electrochemical synthesis of Pd-NPs, Ag-NPs and PB}

To compare the efficiency of Pd-NPs towards peroxide sensing with alternative catalytic systems, electroplated silver, Ag-NPs, and PB nanoparticles were synthesized on the surface of SPE/ GO electrodes. The synthesis of Ag-NPs was performed at $-3 \mathrm{~mA}$ for $60 \mathrm{~s}$ from an $\mathrm{AgCl}$ and $\mathrm{K}_{4}\left[\mathrm{Fe}(\mathrm{CN})_{6}\right]$ containing electrolyte according to the following sequence: ${ }^{25}$

$$
\begin{gathered}
2 \mathrm{AgCl}+\mathrm{K}_{4}\left[\mathrm{Fe}(\mathrm{CN})_{6}\right]=\mathrm{K}_{4}\left[\mathrm{Ag}_{2}(\mathrm{CN})_{6}\right]+\mathrm{FeCl}_{2} \\
\mathrm{FeCl}_{2}+\mathrm{H}_{2} \mathrm{O}+\mathrm{Na}_{2} \mathrm{CO}_{3}=\mathrm{Fe}(\mathrm{OH})_{2}+\mathrm{CO}_{2}+2 \mathrm{NaCl} \\
2 \mathrm{Fe}(\mathrm{OH})_{2}+0.5 \mathrm{O}_{2}+\mathrm{H}_{2} \mathrm{O}=2 \mathrm{Fe}(\mathrm{OH})_{3}
\end{gathered}
$$

The formed $\mathrm{Fe}(\mathrm{OH})_{3}$ was removed by filtration. The electroplating of pure Pd-NPs was performed according to an earlier reported protocol. ${ }^{25}$

For electroplating of PB from an electrolyte containing $0.25 \mathrm{mM} \mathrm{K}_{3} \mathrm{Fe}(\mathrm{CN})_{6}$ and $0.25 \mathrm{mM} \mathrm{FeCl}_{3}$, a recently reported procedure was applied. ${ }^{26}$

\subsection{One-step synthesis of enzymatic nanobiosensors}

One-step fabrication of nanobiosensors with encapsulated GOx or AOx (depending on the nature of the targeted analyte) and Nafion ${ }^{\circledR} 117$ (further referred to as Nafion or Naf) was carried out directly over the surface of SPE/GO $\left(0.12 \mathrm{~cm}^{2}\right) .^{5}$ Briefly, a multiple electrolyte solution containing a Pd-electrolyte, GOx or AOx dissolved in phosphate buffer and Nafion mixed in the ratio of $1: 1: 1 \mathrm{v} / \mathrm{v} / \mathrm{v}$ was prepared and dropped on SPE/GO. Furthermore, a current of $-2.5 \mathrm{~mA}$ was passed through the droplet for $30 \mathrm{~s}$ in galvanostatic mode.

The electroplating of the Ag-NP-based nanobiosensors was carried out from the multiple electrolyte solution containing a Ag-electrolyte, GOx or AOx dissolved in phosphate buffer and Nafion mixed in the volume ratio of $1: 1: 1 \mathrm{v} / \mathrm{v} / \mathrm{v}$. The depo- 
sition was conducted at the current of $-3 \mathrm{~mA}$ for $60 \mathrm{~s}$. Remarkably, the electrodeposition performed from the multiple electrolyte solution resulted in the formation of polymerdoped enzymatic Pd-NPs, Ag-NPs or PB hybrids on SPE/GO. The experiments were carried out on a one-channel biologic Potentiostat PalmSens4 (PalmSens, Utrecht, The Netherlands).

\subsection{Oxygen minisensor studies}

To test the electrochemical behavior of pure electroplated PdNPs, Ag-NPs, PB-NPs and their polymer-doped enzymatic hybrids towards peroxide, different concentrations of hydrogen peroxide were dropped on the surface of modified electrodes. The obtained responses were recorded in cyclic voltammogram (CV) mode from $-0.4 \mathrm{~V}$ to $0.4 \mathrm{~V}$ (unless stated otherwise). The release of oxygen $\left(\mu \mathrm{mol} \mathrm{L} \mathrm{L}^{-1}\right)$ as a product of peroxide decomposition was monitored with an OXR430 retractable needle-type fiber-optic oxygen minisensor (Pyro Science GmbH, Aachen, Germany). The reaction can be summarized as follows (on the electrode): ${ }^{27}$

$$
\mathrm{H}_{2} \mathrm{O}_{2} \rightarrow \mathrm{O}_{2} \uparrow+2 \mathrm{H}^{+}+2 \mathrm{e}^{-}
$$

Notably, the detailed mechanism of this reaction is difficult to determine and depends on the nature of the used NPs. Nevertheless, this sequence clearly demonstrates the reason for oxygen release measured with the oxygen minisensor in the designed systems. ${ }^{27}$

Furthermore, to normalize the rates of oxygen generation to the amount of catalysts, we used a quartz crystal microbalance (QCM-sense) system operated in droplet mode. ${ }^{5}$ The mass of electroplated co-deposits (mass in ng) was calculated using the Sauerbrey equation. ${ }^{5}$

\subsection{Laser desorption ionization mass spectrometry (LDI-MS)}

To ensure enzyme encapsulation together with nanoparticles, LDI-MS screening of the modified SPE/GO surface was used. ${ }^{21}$ LDI-MS investigations were carried out on a Bruker Esquire 3000 + ESI-ion trap MS (Bruker Daltonics, Bremen, Germany) operated using Bruker Esquire control 5.3 software equipped with an atmospheric pressure (AP-MALDI) ion source and $\mathrm{Nd}$ : YAG solid-state laser (355 $\mathrm{nm})$.

\subsection{Electrochemical characterization of enzymatic Pd-NPs- based nanobiosensors}

Hydrogen peroxide $\left(\mathrm{H}_{2} \mathrm{O}_{2}\right)$ is a product of glucose/alcohol oxidation in the presence of oxidases (GOx/AOx) and molecular oxygen. ${ }^{28}$ For example, for glucose oxidation the reaction can be written as follows:

$$
\begin{gathered}
\text { Glucose }+\operatorname{GOx}\left({ }_{\text {ox }}\right) \rightarrow \operatorname{GOx}(\text { red } \\
\operatorname{GOx}\left({ }_{\text {red }}\right)+\mathrm{O}_{2} \rightarrow \operatorname{GOx}\left({ }_{\text {ox }}\right)+\mathrm{H}_{2} \mathrm{O}_{2}
\end{gathered}
$$

and for alcohols:

$$
\mathrm{RCH}_{2} \mathrm{OH}+\mathrm{O}_{2} \stackrel{\mathrm{AOx}}{\longrightarrow} \mathrm{RCHO}+\mathrm{H}_{2} \mathrm{O}_{2}
$$

$\mathrm{H}_{2} \mathrm{O}_{2}$ is an electroactive species which can be reduced or oxidized on the electrode surface depending on the used polar- ization mode. ${ }^{29}$ The electrochemical response of the one-step nanobiosensors was recorded in CV mode on the one-channel biologic Potentiostat PalmSens4 (PalmSens, Utrecht, The Netherlands). The CV curves were obtained by placing a $150 \mu \mathrm{L}$ droplet of pure phosphate buffer or targeted bioanalyte over all three electrodes.

To validate the features of $\mathrm{H}_{2} \mathrm{O}_{2}$ decomposition in diluted solutions, we used a glassy carbon electrode, GCE, in disk form $\left(0.07 \mathrm{~cm}^{2}\right)$ as a working electrode. A set of experiments was performed with a conventional three-electrode electrochemical cell (15 mL) equipped with saturated silver chloride and a platinum wire counter electrode. The inert (argon) or air atmosphere was maintained with Schlenk's line. The layer of Pd-NPs was deposited on the GCE surface from a $1 \mathrm{mM}$ solution of $\mathrm{Pd}\left(\mathrm{NH}_{3}\right)_{4} \mathrm{Cl}_{2}+0.1 \mathrm{M} \mathrm{KNO}_{3}$ under the same conditions as those used for the preparation of Pd-NPs modified SPE/GO. All measurements involving the GCE were carried out on the potentiostat Autolab PGSTAT 101 (Metrohm AG, Germany).

\section{7. pH measurements}

$\mathrm{pH}$ measurements were performed in droplet mode with a HORIBA LAQUATWIN PH-22 pH sensor (MMM tech support GmbH\&Co KG, Berlin, Germany).

\subsection{Scanning electron microscopy (SEM) study}

The morphology of the synthesized nanobiosensors was investigated by scanning electron microscopy on a 400 FEG SEM (Hillsboro, OR, USA). The images were taken in high-resolution mode.

\subsection{RAMAN spectroscopy}

To verify the formation of Pd-oxides on the surface of the nanobiosensors, RAMAN studies were conducted. The experiments were performed under ambient conditions on a HORIBA Jobin Yvon (Longmujeau, France) Raman microscope equipped with an Nd:YAG laser (Melles Griot, IDEX Optics and Photonics, Albuquerque, NM, USA, wavelength of $532 \mathrm{~nm}$ ).

\section{Results and discussion}

\subsection{Superior electrochemical behavior of electroplated Pd- NPs towards $\mathrm{H}_{2} \mathrm{O}_{2}$ sensing}

To choose the most sensitive electrocatalyst in the design of oxidase-associated peroxide oxidation co-catalysis, the analytical merit of Pd-, Ag- and PB-based nanoparticles (NPs), viz. the efficiency towards peroxide sensing, was evaluated with the oxygen minisensor. Comparison of various compositions (Fig. S1 $\dagger$ ) showed a superior behavior of active layers containing Pd-NPs towards $\mathrm{H}_{2} \mathrm{O}_{2}$ in a range of 10-100 mM. The overperformance of the Pd-NPs-based electrocatalysts can be explained in terms of kinetics that is expected to be slower on Ag-NPs than on palladium. Prussian Blue nanoparticles (PB-NPs) are usually used as the electrocatalyst for hydrogen peroxide reduction. Depending on the applied potential, hydrogen peroxide can be reduced on the surface of PB-NPs to 
hydroxide ions that will not affect the dissolved oxygen level in solution. Therefore, the release of oxygen recorded at a high concentration of applied $\mathrm{H}_{2} \mathrm{O}_{2}$ on the sensors containing PB-NPs (Fig. S1 $\dagger$ ) can be explained by the decomposition of hydrogen peroxide on the surface of the SPE/GO electrode. ${ }^{30}$

Notably, the sensing of $\mathrm{H}_{2} \mathrm{O}_{2}$ on Pd-NPs did not show any irreversible changes in the sensor response that is an important criterion for the development of long-term nanobiosensors. Thus, no changes in the electrochemical behavior of PdNPs were detected during the transition from low to high $\mathrm{H}_{2} \mathrm{O}_{2}$ concentration levels and back (see ESI, Fig. S2†).

Hence, the usage of electroplated Pd-NPs as an electrocatalyst in nanobiosensor design can support the efficient sensing of hydrogen peroxide released during specific enzymatic reaction in the presence of a bioanalyte (see section 3.2.2).

\subsection{Electrochemical tuning of Pd-NPs-based nanobiosensors at the cathodic potential}

3.2.1. Competition between the oxygen reduction reaction and hydrogen peroxide sensing on Pd-NPs. A further study of Pd-NPs behavior towards $\mathrm{H}_{2} \mathrm{O}_{2}$ sensing in a micro-analytical concentration range $(1 \mu \mathrm{M}-5 \mathrm{mM})$ was conducted by electrochemical and oxygen minisensor experiments. The electrochemical response was recorded in amperometric mode (AM) at a constant applied potential of $-0.08 \mathrm{~V}^{24}$

Our results indicate a strong competition in the range of $1 \mu \mathrm{M}-5 \mathrm{mM}$ between the oxygen reduction reaction (ORR) and hydrogen peroxide detection on Pd-NPs, Fig. 1.

Briefly, oxygen consumption by Pd-NPs was recorded in the presence of $\mathrm{H}_{2} \mathrm{O}_{2}$ up to a level of $100 \mu \mathrm{M}$. Knowing that in hybrid nanobiosensors the amount of Pd-NPs is at least three times less $(1: 1: 1 \mathrm{v} / \mathrm{v} / \mathrm{v}$ mixing, see the Experimental part) $v s$. pure Pd-NPs (ESI, Fig. S1 $\dagger$ ) and their efficiency to decompose $\mathrm{H}_{2} \mathrm{O}_{2}$ is also several orders of magnitude worse, it is expected that the concentration range of oxygen consumption in the presence of $\mathrm{H}_{2} \mathrm{O}_{2}$ can be readily extended. For example, during the detection of alcohols with Pd-based enzymatic biosensors it was shown that at an applied potential of $-0.08 \mathrm{~V}$ instead of a negative current increase (Fig. 1, see the inset starting from $100 \mu \mathrm{M}$ ) with an increase of loaded analyte concentration (caused by an increase of released hydrogen peroxide as a product of enzymatic reaction), a continuous decrease of the negative current from the hybrid AOx/Nafion/Pd-NPs amperometric biosensors was recorded at least up to the level of $10 \mathrm{mM}^{24}$

To sum it up, the observed strong competition between the ORR and peroxide sensing on Pd-NPs will dramatically affect the electrochemical response and analytical merit of the PdNPs-based nanobiosensors with encapsulated oxidases (see section 3.2.2).

3.2.2. Electrochemical behavior of one-step designed enzymatic Pd-NPs-based nanobiosensors. In a search for an answer to the detected phenomenon (see section 3.2.1), CV studies in a wide scan window (from $-0.5 \mathrm{~V}$ to $0.7 \mathrm{~V}$ ) for Pd-NPs-based nanobiosensors with encapsulated enzymes were performed in buffer solution and in the presence of bioanalytes. Surprisingly, almost no changes in CV curves were recorded for Pd-NPs-based nanobiosensors with encapsulated $\mathrm{AOx}$ or GOx tested in phosphate buffer or in the presence of bioanalytes, i.e. low-molecular weight alcohols (EtOH and $\mathrm{BuOH})$ or glucose, Fig. 2A and B. Thus, the cathodic peak potential at -0.05 to $-0.1 \mathrm{~V}$ attributed to $\mathrm{PdO}$ reduction (the presence of

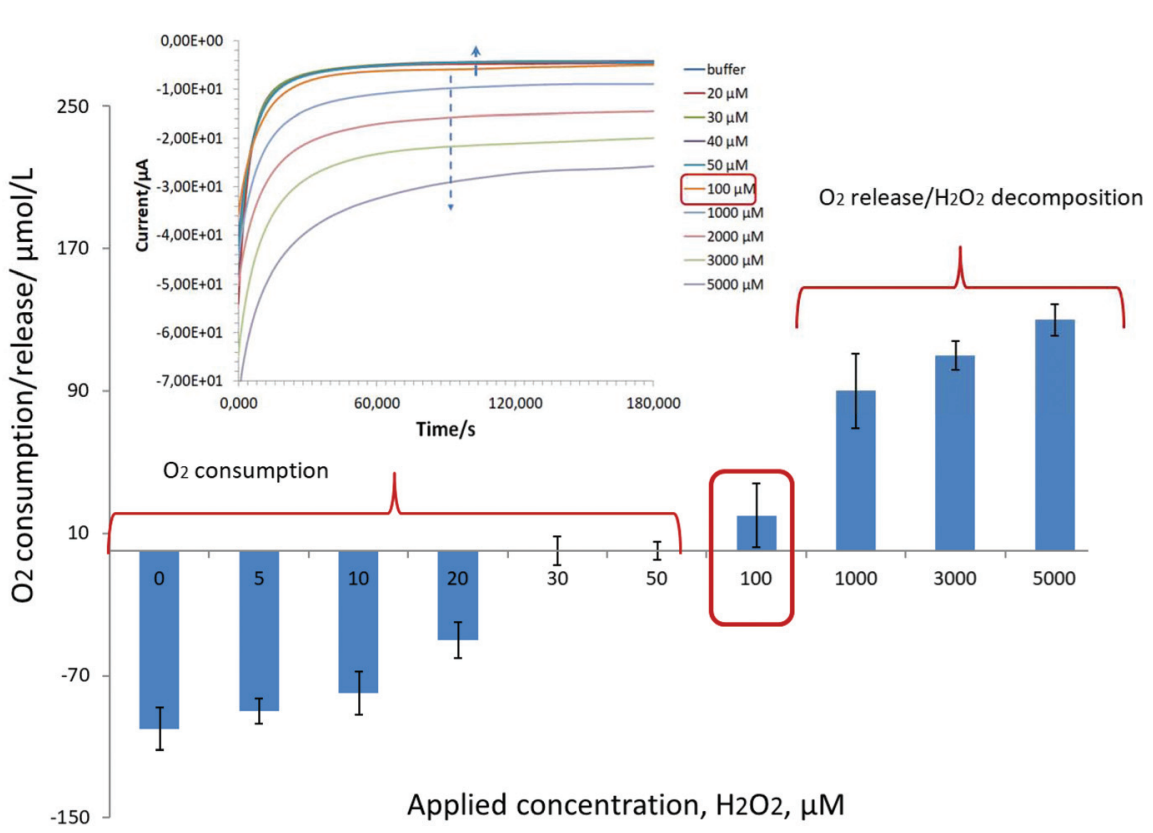

Fig. 1 The response of the optical oxygen microsensor during reaction between pure/intact Pd-NPs (synthesized at $-2.5 \mathrm{~mA}$ for $30 \mathrm{~s}$ ) and hydrogen peroxide (the experiments were performed in triplicate at $20 \pm 2{ }^{\circ} \mathrm{C}$, and the signal was recorded at $100 \mathrm{~s}$ ). Inset - the amperometric responses obtained from Pd-NPs during the detection of $\mathrm{H}_{2} \mathrm{O}_{2}$ at an applied potential of $-0.08 \mathrm{~V}$. 
A

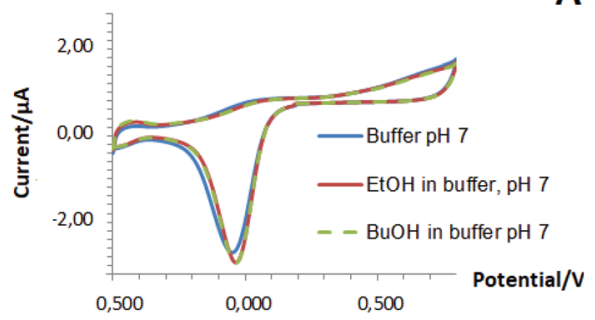

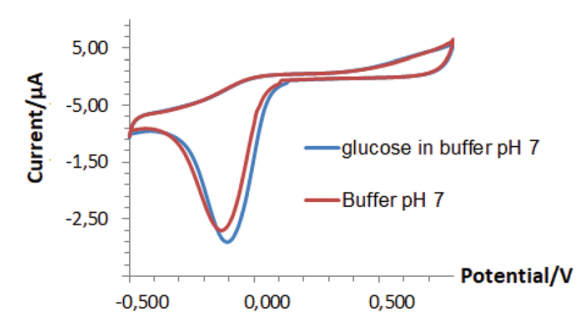
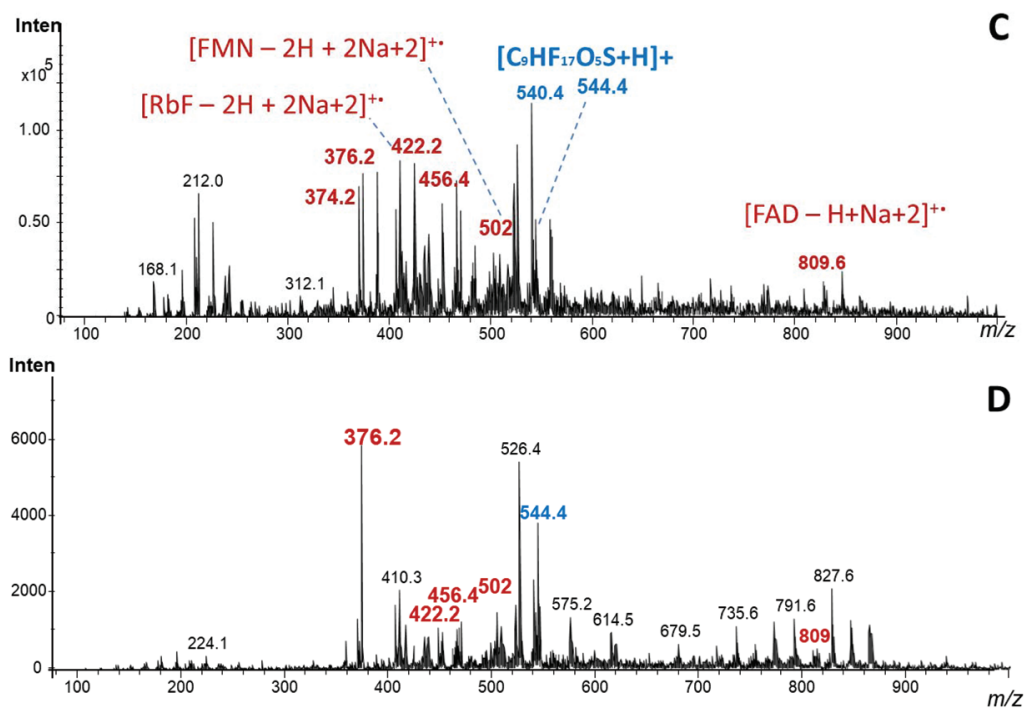

Fig. 2 (A and B) CV curves recorded from the one-step Pd-NPs-based nanobiosensors with encapsulated AOx (A), GOx (B) and Nafion recorded in a phosphate buffer, EtOH $(10 \mathrm{mM})$ and $\mathrm{BuOH}(10 \mathrm{mM})$ at a scan rate of $20 \mathrm{mV} \mathrm{s}^{-1}$ and $\mathrm{pH} 7 \mathrm{vs}$. Ag/AgCl/sat.KCl. (C and D) LDI-MS spectra from PdNPs-based nanobiosensors with encapsulated AOx (C), GOx (D) and Nafion; laser fluence: $45 \%$.

PdO was confirmed by RAMAN study, see ESI, Fig. S3†) and the ORR did not change in the presence of analytes in solution. The cathodic and anodic waves at $-0.45 \mathrm{~V}$ were related to reversible hydrogen adsorption/desorption occurring on palladium. ${ }^{23}$ The increase of the anodic current in the range of 0.4-0.7 $\mathrm{V}$ corresponded to oxygen adsorption $\left(\mathrm{Pd}_{x} \mathrm{O}_{y}\right.$ formation). ${ }^{29}$

At the same time, the presence of enzymes (evaluated as a co-factor, i.e. flavin adenine dinucleotide, $\mathrm{FAD}$ ) in the structure of the one-step designed Pd-NPs-based nanobiosensors and the efficiency of the performed co-deposition procedure was confirmed by LDI-MS, Fig. 2C and D. Thus, in addition to Nafion $(\mathrm{m} / \mathrm{z}$ at 544$), \operatorname{FAD}(\mathrm{m} / \mathrm{z}$ at 809$)$ as a small molecular weight organic compound, its related fragments (FMN and RbF) and their adducts were clearly seen in mass spectra obtained from the one-step nanobiosensors with encapsulated oxidases. ${ }^{22}$

In other words, despite the obvious presence of enzymes in the end-structure of the Pd-NPs-based nanobiosensors, their operation at the cathodic range of potentials does not seem to be a trivial task. This is in particular true if the hydrogen peroxide concentration is comparable with the concentration of dissolved oxygen (see Fig. 1).
It appears that multiple reactions simultaneously occurring on the surface of Pd-NPs (see section 3.2.1) severely affect the responses supplied by the one-step designed nanobiosensors with encapsulated oxidases. This hypothesis was approved through the standard response studies performed in AM mode at the constant applied potential of $-0.05 \mathrm{~V}$ with a new droplet of buffer spotted on the surface of the one-step Pd-NPs-based nanobiosensor with encapsulated AOx and Nafion (ESI, Fig. S4†). Briefly, a continuous decrease of the cathodic current in a cycle experiment from droplet to droplet was observed indicating a high instability of the sensor's basic line. This effect can be explained by Pd-oxide formation/ reduction (ESI, Fig. S3†) on the one side and by the ORR on the other side (see section 3.3). This type of electrochemical behavior is an illustration of the general problem of oxidasebased biosensors: the dependence on oxygen concentration, which might be quite uncertain in a complex system, viz. hybrid Pd-NPs-based enzymatic nanobiosensors.

Furthermore, we conducted a blank experiment with pure Pd-NPs deposited on the surface of SPE/GO under different electroplating conditions (deposition time), tested their response in a conventional $\mathrm{CV}$ mode, and compared their per- 


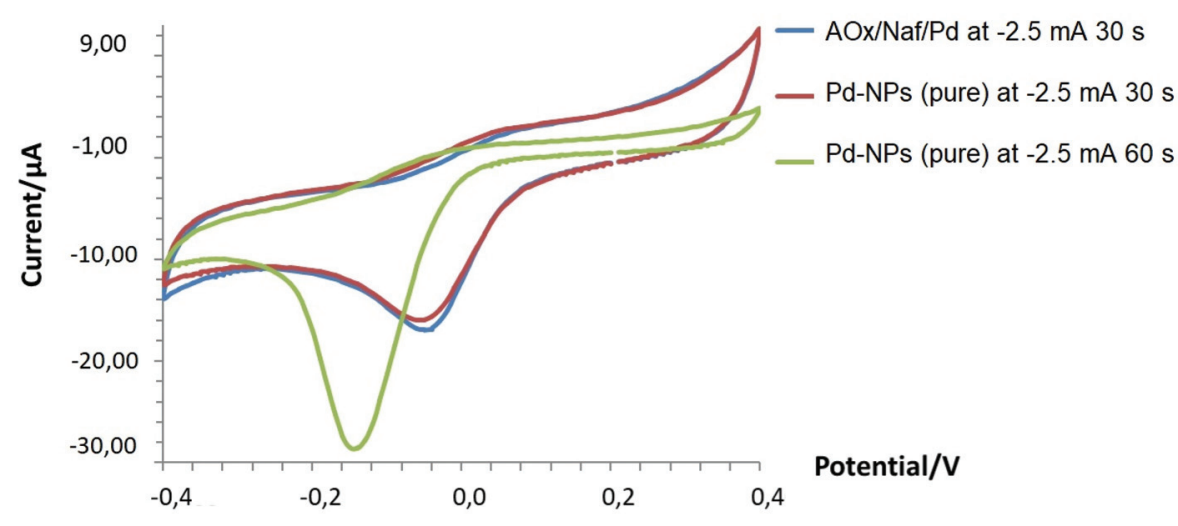

Pd-NPs, $-2.5 \mathrm{~mA}, 30 \mathrm{~s}$

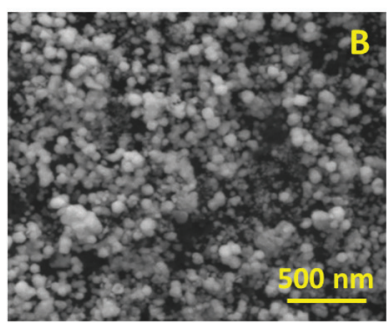

Pd-NPs, $-2.5 \mathrm{~mA}, 60 \mathrm{~s}$

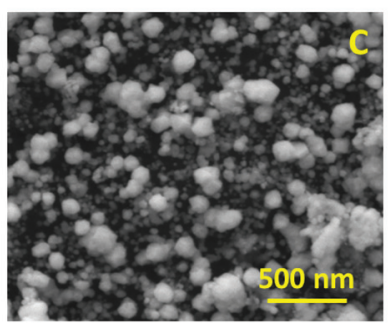

$\mathrm{AOx} /$ Nafion/Pd $-2.5 \mathrm{~mA}, 30 \mathrm{~s}$

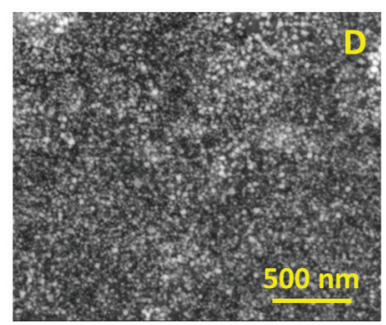

Fig. 3 Top - CV curves (A) recorded in phosphate buffer at a scan rate of $20 \mathrm{mV} \mathrm{s}^{-1}$ from pure/intact Pd-NPs produced at different deposition times and their hybrid (shown for AOx/Nafion/Pd) vs. $\mathrm{Ag} / \mathrm{AgCl} / \mathrm{sat}$. $\mathrm{KCl}$. Bottom - SEM images of pure/intact Pd-NPs (B and C) and the one-step PdNPs-based hybrid nanobiosensor (D).

formance with that of an AOx/Nafion/Pd system. As can be seen from the CV curves (Fig. 3A), even in the absence of an enzyme, pure Pd-NPs produced at $-2.5 \mathrm{~mA}$ for $30 \mathrm{~s}$ showed the same type of electrochemical behavior at the cathodic branch as was observed for one-step designed AOx/Nafion/Pd synthesized under the same conditions. However, the peak potential in the cathodic range of $\mathrm{CV}$ for larger particles $(-150 \mathrm{mQ}$, $-2.5 \mathrm{~mA}$ for $60 \mathrm{~s})$ shifted towards a more negative range $v s$. smaller Pd-NPs $(-75 \mathrm{mQ},-2.5 \mathrm{~mA}$ for $30 \mathrm{~s})$. We assume that this effect is caused by the changes in the surface density and size of the Pd-NPs (Fig. 3B and C) that can impact the position of the cathodic peak and amount of the formed PdO. The presence of the polymer and enzyme in the one-step AOx/Naf/Pd nanobiosensor leads to diminution of the end-particle size as compared to pure Pd-NPs synthesized under the same conditions; see Fig. 3D.

In addition, a strong dependency of the cathodic peak position on temperature and solution acidity/basicity was revealed (see ESI, Fig. S5†). This means that the environmental and storage conditions will severely affect the surface chemistry of the Pd-NPs-based nanobiosensors and the amount of the formed palladium oxides. Hence, the one-step designed nanobiosensors with encapsulated enzymes cannot be reliably operated at the cathodic range of potentials. Therefore, the development of a read-out platform and operational guidelines of one-step designed Pd-NPs-based amperometric nanobiosensors is highly desirable (see the next section).

\subsection{Development of operational principles of Pd-NPs-based nanobiosensors and their validation with a conventional three-electrode electrochemical cell}

To verify the impact of palladium oxides ${ }^{29}$ on the electrochemical read-out supplied by Pd-NPs, CV studies were conducted for the Pd-NPs-modified GCE under an Ar-atmosphere in the absence of $\mathrm{O}_{2}$ and in the presence of oxygen at different anodic limits, Fig. S6. $\dagger$

The obtained data indicate the increase of both anodic and cathodic currents with the increase of the anodic polarization limit. This means that the presence of molecular oxygen and extension of the anodic polarization limit facilitate palladium oxide formation resulting in the increase of oxygen electroreduction during the backward scan. ${ }^{29}$ It is important to mention that the cathodic peak is increased in the presence of dissolved oxygen due to the ORR.

Furthermore, to establish the polarization mode where hydrogen peroxide can be selectively and reliable detected by intact Pd-NPs or their enzyme-containing hybrids in the presence of dissolved oxygen, several preliminary tests were performed in a classic three-electrode electrochemical cell with a GCE electrode (see section 2.6). 

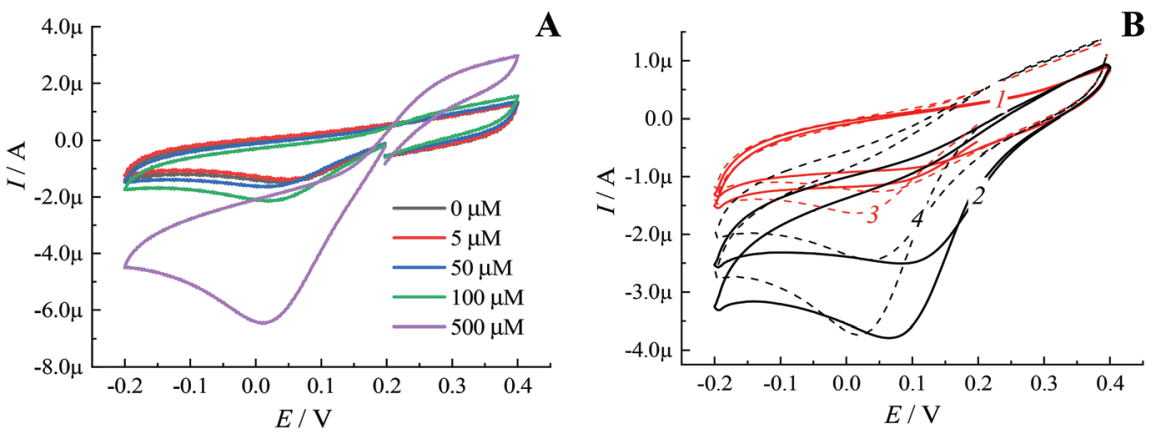

Fig. $4 \mathrm{CV}$ responses of the Pd-NPs-modified GCE recorded at a scan rate $50 \mathrm{mV} \mathrm{s}^{-1}$ in phosphate buffer (A) with various $\mathrm{H}_{2} \mathrm{O}_{2}$ concentration at $\mathrm{pH}$ 7.5 (the experiments were performed under an inert atmosphere) and in $50 \mu \mathrm{M} \mathrm{H}_{2} \mathrm{O}_{2}$ (B) at different pH values: $\mathrm{pH} 7.5$ (1 and 2) and pH 5.5 (3 and 4) under ( 1 and 3 ) inert and ( 2 and 4$)$ air atmospheres.

Notably, in solutions with a low concentration of hydrogen peroxide even under an inert atmosphere almost no sensitivity of the cathodic peak current to the presence of $\mathrm{H}_{2} \mathrm{O}_{2}$ was observed (Fig. 4A). These results were in line with the dependency revealed by oxygen minisensor studies performed for SPE/GO modified by Pd-NPs (see Fig. 1). Due to the ORR, the presence of molecular oxygen in solution leads to an increase in the cathodic peak current. In addition, the cathodic peak potential is shifted depending on $\mathrm{pH}$ (Fig. 4B). Since the concentration of dissolved oxygen strongly depends on the air content and temperature, the cathodic peak current in oxygenated solutions cannot be used to detect hydrogen peroxide with Pd-NPs-based sensors regardless of the used electrode, i.e. SPE/GO or GCE.

In contrast, the current of the anodic branch at a potential of $0.2-0.4 \mathrm{~V}$ grows depending on the peroxide concentration due to hydrogen peroxide oxidative decomposition occurring on the Pd surface. Thus, peroxide decomposition facilitates the formation of palladium oxides resulting in the increase of the anodic current in the above-mentioned range of potentials.

Thus, it appears possible to quantitatively detect peroxide in oxygenated aqueous solutions using Pd-NPs-modified electrodes operated at the anodic potentials. However, to refresh the electrode surface, it is highly necessary to reduce Pd-oxides formed during anodic polarization. For this goal, we optimized the following double step procedure: the first step is a polarization at the cathodic peak potential to reduce PdO, and the second step is a polarization at the anodic potential in a range of $0.2-0.4 \mathrm{~V}$ to detect/record the current related to hydrogen peroxide decomposition.

Importantly, the potential and duration of the first polarization step depend on the $\mathrm{pH}$ of a solution (buffer or fermentation media), the presence of molecular oxygen, intact Pd-NPs size and the nature of organic/bioreceptor compounds in the structure of the functional sensing layer. In this regard, the polarization step performed at the cathodic peak potential should be preliminarily estimated by $\mathrm{CV}$ studies for various experimental conditions.

For example, for phosphate buffer with $\mathrm{pH} 7.5$ and $\mathrm{pH} 5.5$ the multi-step amperometric procedure on the Pd-NPs-modi- fied GCE was carried out as follows: the polarization duration at the cathodic peak potential was set to $120 \mathrm{~s}$, and the polarization duration at an anode potential of $0.4 \mathrm{~V}$ was $30 \mathrm{~s}$. As is seen from Fig. S7 (ESI), $\dagger$ the current of the second polarization step is strongly proportional to the concentration of the dissolved hydrogen peroxide in solution. At the same time, the change in $\mathrm{pH}$ does not significantly affect the current responses at the anodic polarization potentials. More importantly, the presence of molecular oxygen in solutions with low $\mathrm{H}_{2} \mathrm{O}_{2}$ concentration has a little impact on the current response; see ESI, Fig. S7.†

Following a similar multi-step polarization procedure as described above, the response of Pd-NPs-modified SPE/GO towards hydrogen peroxide detection at the anodic potential range was validated; see ESI, Fig. S8. $\dagger$ Notably, the electrochemical read-out mode will significantly impact the analytical performance of the Pd-NPs-based amperometric nanobiosensors (see the next section).

\subsection{Analytical performance of Pd-NPs-based amperometric nanobiosensors at the anodic potentials}

3.4.1. $\mathrm{H}_{2} \mathrm{O}_{2}$ sensing. Apart from the established optimal electrochemical operational mode, the behavior of Pd-NPsbased amperometric sensors for non-enzymatic hydrogen peroxide detection has been reported to be strongly dependent on the mechanical stability of NPs, their density and coverage rate of the electrode surface in the cathodic branch of $\mathrm{CV} .^{5}$

Next, the response of the Pd-NPs synthesized at different electrodeposition parameters (shown for $30 \mathrm{~s}$ and $60 \mathrm{~s}$ ) to hydrogen peroxide detection was evaluated in the optimized multi-step polarization mode. Significantly, the electrocatalytic ability of Pd-NPs-modified SPE/GO changes in the anodic range with Pd-NPs density and size; see ESI, Fig. S9. $\dagger$ Thus, the increase of deposition time from $30 \mathrm{~s}$ to $60 \mathrm{~s}$ was accompanied by an increase of NPs size from $20 \mathrm{~nm}$ to 70-75 nm (see also Fig. 3B and C) followed by the subsequent decrease in $\mathrm{H}_{2} \mathrm{O}_{2}$ sensitivity.

Furthermore, the tuning of the analytical performance of Pd-NPs towards $\mathrm{H}_{2} \mathrm{O}_{2}$ detection and comparison of their analytical merits at different electrochemical operational modes were performed for nanoparticles produced at $-2.5 \mathrm{~mA}$ 
Table 1 The impact of electrochemical operational mode on the analytical performance of Pd-NPs $(-2.5 \mathrm{~mA}$, deposition time: $30 \mathrm{~s})$ during $\mathrm{H}_{2} \mathrm{O}_{2}$ detection

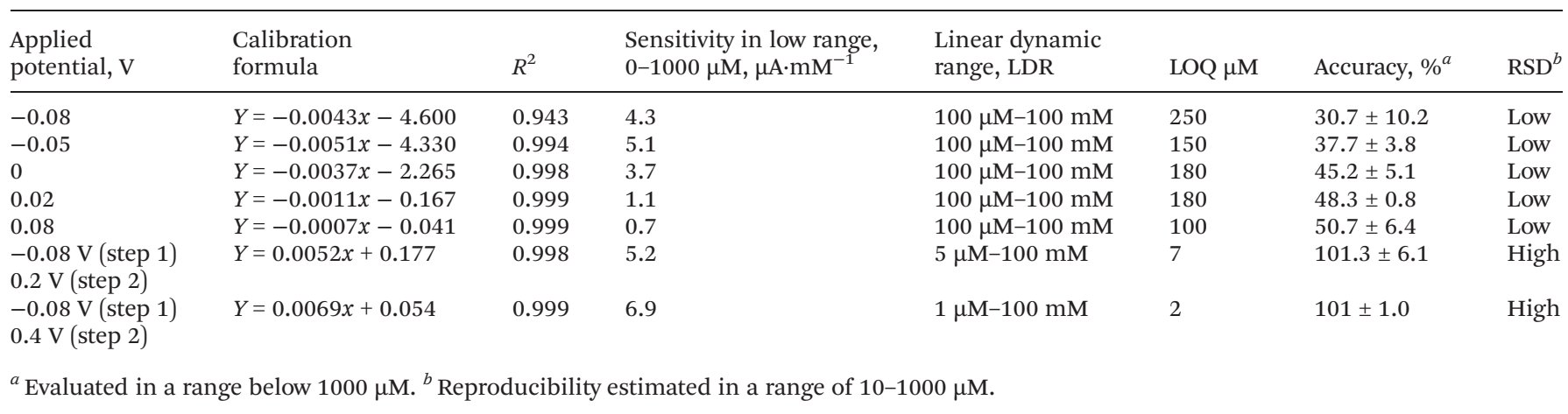

for $30 \mathrm{~s}$. The results of this tuning are summarized in Table 1. Obviously, advanced analytical merits of this sensing system can be achieved by the optimization of the read-out platform delivered by Pd-NPs.

Despite the higher sensitivity of Pd-NPs $(-2.5 \mathrm{~mA}, 30 \mathrm{~s})$ to $\mathrm{H}_{2} \mathrm{O}_{2}$ detection revealed at $0.4 \mathrm{~V}$ vs. $0.2 \mathrm{~V}$, polarization at $0.2 \mathrm{~V}$ should be considered in further experiments to minimize the interferences increasing at $0.4 \mathrm{~V}$ from other electroactive species (acetophenone, acetic acid, ascorbic acid, etc.) possibly present in the real samples.

Furthermore, during the validation of the analytical performance of Pd-NPs for $\mathrm{H}_{2} \mathrm{O}_{2}$ detection, the accuracy test at a cathodic polarization potential of $-0.08 \mathrm{~V}$ held for $30 \mathrm{~s}$ followed by signal read-out at $0.2 \mathrm{~V}$ was performed in multi-step amperometric mode (MAM), Fig. 5. In addition to the excellent accuracy detected at $0.2 \mathrm{~V}$, an advanced basic line stability and tolerance to the presence of interfering electroactive species were observed for Pd-NPs operated at the optimized read-out MAM mode; see ESI, Fig. S10-S12. $\dagger$

Thus, by gaining closer insights into the designed electrochemical system and by a detailed study of the fundamental processes affecting the generated signal, it was possible to establish and validate the operational conditions of the hybrid Pd-NPs-based nanobiosensors.

3.4.2. Enzymatic sensing by Pd-NPs-based nanobiosensors. Based on the obtained dependencies, the performance of oxidase-associated peroxide oxidation Pd-NPs was evaluated in one-step designed nanobiosensors (with encapsulated GOx as a case study) by optimized the MAM read-out mode. Briefly, the excellent current-concentration dependency was obtained for the one-step designed amperometric Pd-NPs-based nanobiosensor with GOx in an analytical range of glucose, i.e. 1-10 mM (Fig. 6A).

To improve the linear dynamic range of targeted bioanalyte detection (shown for glucose as a case study) by Pd-NPs-based enzymatic nanobiosensors operated at MAM mode, the following modification step to the intact sensor design was implemented. First, a layer of pure Pd-NPs was deposited on the surface of the SPE/GO electrode at $-2.5 \mathrm{~mA}$ for $30 \mathrm{~s}$ in the manner shown in section 3.4.1 (step 1). Next, the encapsulation of GOx with Nafion from the multiple Pd-electrolyte solu-

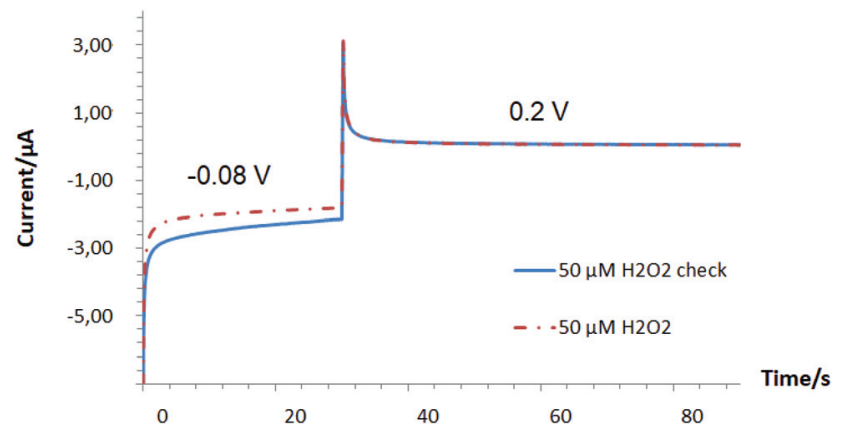

Fig. 5 Electrochemical responses obtained from pure/intact Pd-NPs $(-2.5 \mathrm{~mA}, 30 \mathrm{~s})$ towards $\mathrm{H}_{2} \mathrm{O}_{2}$ detection in the multi-step amperometry mode (MAM): polarization at $-0.08 \mathrm{~V}$ followed by read-out at $0.2 \mathrm{~V}$.

tion (step 2) via one-step deposition with the same electroplating parameters was conducted (will be referred to as two-step designed Pd-NPs-based nanobiosensors). Interestingly, due to the pre-modification step 1 being performed we managed to enhance the linear dynamic range of the proposed glucose biosensing system from 1-10 mM to 1-30 mM, Fig. 6B.

We hypothesize that the first Pd-NPs layer (step 1) allows the increase of the entire electroactive surface area to detect a high concentration of hydrogen peroxide released during enzymatic reaction and, probably, facilitates the electric connection with the Pd-NPs electrocatalyst deposited in the hybrid enzyme contained layer (step 2). Hence, this altogether will help improve the enzymatic activity of the nanobiosensor and significantly improve its overall analytical performance.

Comparison of the analytical merits between the obtained results and data discussed in the literature ${ }^{31-34}$ on peroxidebased glucose biosensors indicates that the proposed MAM procedure for $\mathrm{H}_{2} \mathrm{O}_{2}$ detection permits:

- reduction of the anodic polarization potential value;

- exclusion of the impact of the interfering component (oxygen) on the analytical response of the system;

- enhancement of the linear dynamic range and LOQ;

- improvement of the accuracy and reproducibility of the analysis. 


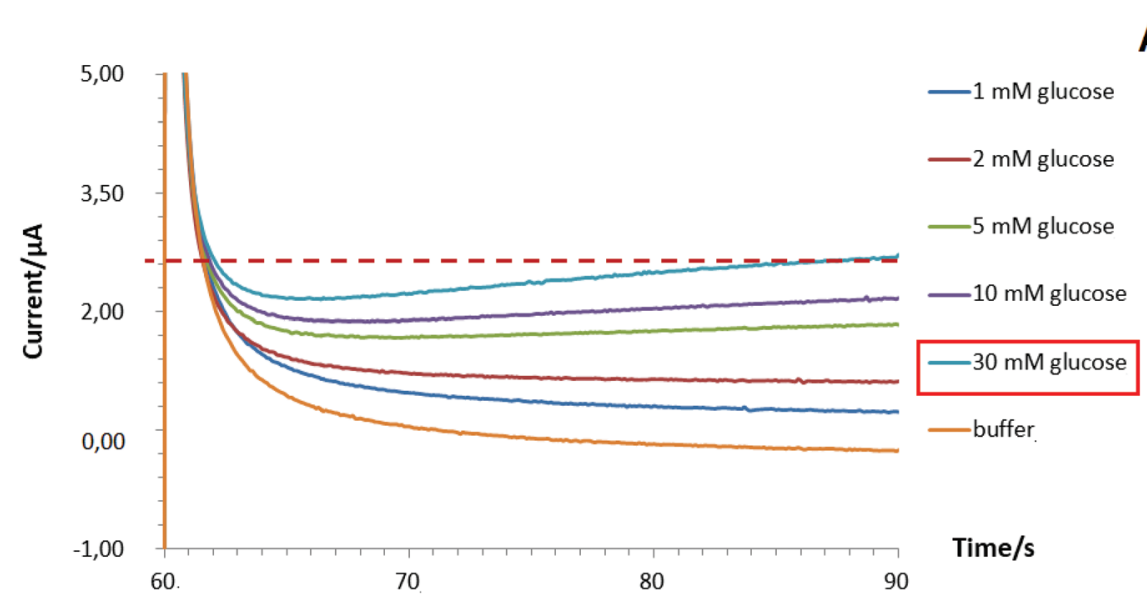

A

B

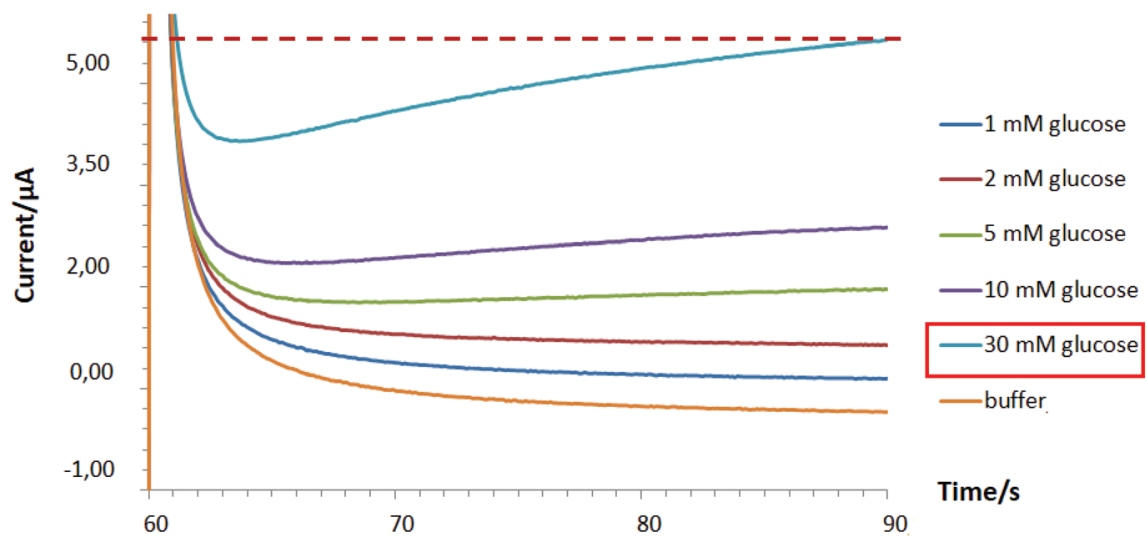

Fig. 6 Chronoamperometric curves recorded in MAM mode from the Pd-NPs-based nanobiosensors with encapsulated GOx (as a case study) in the presence of glucose: (A) one-step designed GOx/Naf/Pd nanobiosensor; and (B) two-step designed GOx/Naf/Pd nanobiosensor.

\section{Conclusions}

Here, a fundamental study on the search and validation of the optimal operating electrochemical parameters of Pd-NPsbased amperometric nanobiosensors was carried out. The role of palladium surface oxides and adsorbed oxygen on the sensitivity and product selectivity in Pd-NPs-based nanobiosensors was confirmed.

As a solution, a novel multi-step amperometric read-out mode (the first step is a polarization at the cathodic peak potential to reduce $\mathrm{PdO}$, and the second step is a polarization at the anodic potential to detect the $\mathrm{H}_{2} \mathrm{O}_{2}$ oxidation current) that results in a faster reduction of $\mathrm{PdO}$, and reliable, sensitive and selective peroxide sensing was developed. The proposed procedure was fully validated in a conventional three-electrode electrochemical cell under both air and inert atmospheres. Finally, re-design of Pd-NPs allowed us to improve the analytical performance of the Pd-NPs-based nanobiosensors that can be readily considered as a possible alternative to sophisticated, expensive, time- and source-consuming enzyme-related approaches (i.e. amount of enzyme, variation of $\mathrm{pH}$ and enzyme strains, immobilization strategies, etc.).
We believe that our results will thus contribute to the future development of noble metal NPs-based nanobiosensors, and also will provide an opportunity to tune their performance based on the requirements of the analytical task.

\section{Author contributions}

Y. E. Silina - conceptualized the concept of the research, developed the one-step nanobiosensor approach, was responsible for the design of the manuscript and data analysis, wrote, reviewed, and edited the manuscript, and handled funding acquisition and project administration. N. Apushkinskaya conducted sensor-related experiments (SPE/GO system), data visualization, validation and formal analysis. N. V. Talagaeva was responsible for electrochemical experiments utilizing the three-electrode electrochemical cell. M. G. Levchenko - performed sample preparation and took part in electrochemical experiments utilizing the three-electrode electrochemical cell. E. V. Zolotukhina - was responsible for the conceptualization and design of electrochemical experiments, validation 
strategies, data analysis, writing, reviewing, and editing of the manuscript.

\section{Conflicts of interest}

There are no conflicts to declare.

\section{Acknowledgements}

This study was a part of the research program of Y. E. S. funded by the Deutsche Forschungsgemeinschaft (DFG, German Research Foundation, project 427949628). The authors would like to thank the German-Russian Interdisciplinary Science Center (G-RISC) for the support of collaboration between the University of Saarland (Germany) and Institute of Problems of Chemical Physics RAS (Moscow region, Russia). The authors N. V. Talagaeva, M. G. Levchenko and E. V. Zolotukhina performed their work within the framework of the State assignment (number AAAA-A19119061890019-5). Y. E. S. also thanks Dr Marcus Koch (INM Leibniz Institute of New Materials, Saarbrücken, Germany) for the performed SEM studies of Pd-NPs-based nanostructures and Prof. Dr Bruce Morgan (University of Saarland) for continuing support of this research at the department.

\section{References}

1 A. Vasilescu, S. Andreescu, C. Bala, S. C. Litescu, T. Noguer and J. L. Marty, Biosens. Bioelectron., 2003, 18, 781-790.

2 A. M. Titoiu, G. Necula-Petrareanu, D. Visinescu, V. Dinca, A. Bonciu, C. N. Mihailescu, C. Purcarea, R. Boukherroub, S. Szunerits and A. Vasilescu, Microchim. Acta, 2020, 187(10), 550.

3 A. A. Karyakin, Electroanalysis, 2001, 13, 1-7.

4 A. A. Karyakin and E. E. Karyakina, Sens. Actuators, B, 1999, 57, 268-273.

5 D. Semenova, K. V. Gernaey, B. Morgan and Y. E. Silina, Analyst, 2020, 145, 1014-1024.

6 J. Wang, Microchim. Acta, 2012, 177, 245-270.

7 C. Shan, H. Yang, D. Han, Q. Zhang, A. Ivaska and L. Niu, Biosens. Bioelectron., 2010, 25, 1070-1074.

8 S. Azzouzi, L. Rotariu, A. M. Benito, W. K. Maser, M. Ben Ali and C. Bala, Biosens. Bioelectron., 2015, 15(69), 280-286.

9 J. Bai, Y. Li, J. Du, S. Wang, J. Zheng, Q. Yang and X. Chen, Mater. Chem. Phys., 2007, 106, 412-415.

10 S. H. Kim, G. H. Jeong, D. Choi, S. Yoon, H. B. Jeon, S. M. Lee and S. W. Kim, J. Colloid Interface Sci., 2013, 389(1), 85-90.
11 H. S. Al-Ghamdi and W. E. Mahmoud, Mater. Lett., 2013, 105, 62-64.

12 R. Batool, A. Rhouati, M. H. Nawaz, A. Hayat and J. L. Marty, Biosensors, 2019, 9, 46.

13 M. Holzinger, A. Le Goff and S. Cosnier, Front. Chem., 2014, 2, 1-10.

14 N. Stasyuk, G. Gayda, H. Klepach and M. Gonchar, Sens. Lett., 2016, 14, 1169-1177.

15 N. Tujunen, E. Kaivosoja, V. Protopopova, J. J. ValleDelgado, M. Österberg, J. Koskinen and T. Laurila, Mater. Sci. Eng., C, 2015, 55, 70-78.

16 N. Dimcheva, Curr. Opin. Electrochem., 2020, 19, 35-41.

17 V. Mazeiko, A. Kausaite-Minkstimiene, A. Ramanaviciene, Z. Balevicius and A. Ramanavicius, Sens. Actuators, B, 2013, 189, 187-193.

18 R. D. O'Neill, S. C. Chang, J. P. Lowry and C. J. McNeil, in Biosensors and Bioelectronics, 2004.

19 M. Topçu Sulak, Ö. Gökdoğan, A. Gülce and H. Gülce, Biosens. Bioelectron., 2006, 15(21), 1719-1726.

20 Z. Wen, S. Ci and J. Li, J. Phys. Chem. C, 2009, 113, 1348213487.

21 Y. E. Silina and B. Morgan, Talanta, 2020, 223, 121688.

22 E. V. Butyrskaya, N. Korkmaz, E. V. Zolotukhina, V. Krasiukova and Y. E. Silina, Analyst, 2021, 146, 21722185.

23 R. Rahul, R. K. Singh, B. Bera, R. Devivaraprasad and M. Neergat, Phys. Chem. Chem. Phys., 2015, 17, 1514615155.

24 D. Semenova, T. Pinto, M. Koch, K. V. Gernaey and H. Junicke, Biosens. Bioelectron., 2020, 170, 112702.

25 Y. E. Silina, F. Meier, V. A. Nebolsin, M. Koch and D. A. Volmer, J. Am. Soc. Mass Spectrom., 2014, 25, 841-851.

26 V. B. Isfahani, H. R. Dizaji, N. Memarian and A. Arab, Mater. Res. Express, 2019, 6, 096449.

27 S. B. Hall, E. A. Khudaish and A. L. Hart, Electrochim. Acta, 1998, 43, 579-588.

28 K. Pontius, D. Semenova, Y. E. Silina, K. V. Gernaey and H. Junicke, Front. Bioeng. Biotechnol., 2020, 8, 1-15.

29 K. V. Gor'kov, N. V. Talagaeva, S. A. Kleinikova, N. N. Dremova, M. A. Vorotyntsev and E. V. Zolotukhina, Electrochim. Acta, 2020, 345, 136164.

30 R. S. Ribeiro, A. M. T. Silva, J. L. Figueiredo, J. L. Faria and H. T. Gomes, Carbon, 2013, 62, 97-108.

31 X. B. Sun and Z. F. Ma, Advanced Materials Research, Trans Tech Publications Ltd., 2013, vol. 643, pp. 162-165.

32 Md. M. Rahman, A. J. S. Ahammad, J.-H. Jin, S. J. Ahn and J.-J. Lee, Sensors, 2010, 10, 4855-4886.

33 D. Xiang, L. Yin, J. Ma, E. Guo, Q. Li, Z. Li and K. Liu, Analyst, 2015, 140(2), 644-653.

34 Z. Lu, L. Wu, X. Dai, Y. Wang, M. Sun, C. Zhou, H. Du and H. Rao, J. Hazard. Mater., 2021, 402, 123774. 